\title{
INFLUÊNCIA DO GÊNERO NA DEMANDA VISUAL POR MAPAS EM ESCALAS DIFERENTES EM SISTEMA DE NAVEGAÇÃO E GUIA DE ROTA
}

The gender effect on visual demand for maps at different scales in route guidance and navigation system

\author{
ANA PAULA MARQUES RAMOS ${ }^{1}$ \\ MÔNICA MODESTA SANTOS DECANINI ${ }^{2}$ \\ EDMUR AZEVEDO PUGLIESI ${ }^{3}$ \\ VILMA MAYUMI TACHIBANA ${ }^{4}$
}

\author{
Universidade Estadual Paulista - UNESP \\ ${ }^{1}$ Programa de Pós-Graduação em Ciências Cartográficas \\ ${ }^{2,3}$ Departamento de Cartografia; ${ }^{4}$ Departamento de Estatística
}

R. R. Simonsen, 305 CEP 19060-900 - Pres. Prudente - São Paulo - Brasil marques.engcart@gmail.com; monca@ fct.unesp.br; edmur@fct.unesp.br;

vilma@fct.unesp.br

\section{RESUMO}

Este trabalho investiga a influência do gênero do motorista na demanda visual por mapas dinâmicos exibidos em diferentes escalas em Sistemas de Navegação e Guia de Rota em Automóvel- SINGRA. Um grupo de 26 homens e 26 mulheres participou de um teste realizado em um simulador de direção de baixo custo. A tarefa do motorista consistiu em realizar uma viagem por uma rota desconhecida, auxiliado por um protótipo de SINGRA que exibe mapas em duas diferentes escalas. Este trabalho replica os achados da literatura a respeito de que há uma relação significativa entre o gênero e o desempenho em tarefas visuo-espacial. Os resultados mostram que motoristas de diferentes gêneros apresentam diferentes níveis de demanda visual, tanto para a variação da escala do mapa quanto para a variação da complexidade da manobra. Estes resultados são discutidos com base nas diferenças individuais em termos de habilidade espacial e ansiedade espacial.

Palavras chaves: Sistema de Navegação; Guia de Rota em Automóvel; Escalas; Gênero; Demanda Visual. 


\section{ABSTRACT}

This work investigates the gender effect on visual demand of drivers for dynamic maps at different cartographic scales presented In-Vehicle Route Guidance and Navigation System (RGNS). A group of 52 subjects (26 males and 26 females) took part in an experiment performed in a low-cost driving simulator. the driver's task consisted of navigating in an unknown route using a RGNS prototype which presents maps at two different cartographic scales. This paper replicates the known phenomenon of significant relationships between gender and performance at visualspatial tasks issue. Our results show that drivers of different genders present distinct levels of visual demand both due to the cartographic scales and maneuver complexity variation. These discussed results are based upon individual differences in terms of spatial ability and spatial anxiety.

Keywords: Navigation System; In-vehicle Route guidance; Scales; Gender; Visual Demand.

\section{INTRODUÇÃO}

A análise do movimento dos olhos é um método tradicionalmente aceito para determinar a demanda visual por uma interface e, assim, estudar os problemas de usabilidade no contexto da Interação Homem-Máquina (Human Computer Interaction - HCI) (TSIMHONI et al., 1999; POOLE \& BALL, 2005). Os Sistemas de Navegação e Guia de Rota em Automóvel (SINGRA) são alguns desses tipos de interfaces utilizados dentro dos automóveis para oferecer maior mobilidade e conforto aos motoristas na tarefa de navegação, porém que requerem demanda visual associada à tarefa de direção (KABER et al., 2012). A análise de demanda visual é utilizada como uma medida de avaliação da eficiência de uma interface (POOLE \& BALL, 2005). No contexto de SINGRA, esta medida é adotada para compreender as implicações que a leitura de mapa, com o automóvel em movimento, resulta no desempenho do motorista na tarefa de direção (LABIALE, 2001; PUGLIESI et al., 2009, CHING-TORNG et al., 2010).

Um dos desafios atuais para a área de HCI é melhorar a qualidade dos mapas de navegação utilizados por motoristas de diferentes grupos (ex.: gênero, idade, etc.) e (ou) com distintas características individuais (ex.: habilidade espacial, formação educacional, etc.) (PUGLIESI et al., 2009; LAVIE et al., 2011; LAVIE \& ORONGILAD, 2013; RAMOS et al., 2014b). Dentre as importantes vertentes de pesquisa na área de HCI, destaca-se a seleção da escala dos mapas de SINGRA (UANG \& HWANG, 2003; HO \& LI, 2004; DILLEMUTH, 2007; LEE et al., 2008; SHELEIBY et al., 2008; WU \& ZHANG, 2009; BINFENG et al., 2012; MARQUES et al., 2012; RAMOS et al. 2014c). O intuito das investigações é aumentar a eficiência do motorista no processo de leitura de mapa, sem comprometer seu desempenho na tarefa de direção do automóvel.

A necessidade de se considerar as características individuais e de grupo no projeto de interfaces decorre do fato de que tais características influenciam no desempenho dos usuários ao realizarem determinadas tarefas, principalmente, as de 
caráter visuo-espacial (KOZLOSWKI \& BRYANT, 1977; DABBS et al., 1998; MONTELLO et al., 1999; COLUCCIA et al., 2007; WILKENING \& FABRIKANT, 2011). Diante dessas evidências, os usuários de SINGRA que interagem com mapas durante a direção do automóvel não deveriam ser considerados um grupo homogêneo; pelo contrário, suas características individuais e de grupo deveriam ser levadas em consideração no projeto desses sistemas.

O gênero é uma importante característica de grupo para o contexto de uso de um SINGRA, visto que o número de motoristas mulheres cresceu significativamente nos últimos anos (WAKABAYASHI, 2011), e isso pode diversificar a forma como os mapas são utilizados na navegação em automóvel. Mundialmente, as mulheres são responsáveis pela venda de 30 milhões de automóveis por ano (FREE PRESS, 2013). Apenas nos EUA, as mulheres são responsáveis pela compra de 54\% dos automóveis vendidos anualmente e influenciam em mais de $80 \%$ nas vendas (FLEMING, 2013). Pei-Chun e Shang-I (2013) afirmam que a mudança no segmento de compradores de automóveis é um fator que deve ser considerado no projeto de interfaces que serão utilizadas para apoiar a tarefa de navegação.

Slocum et al. (2001) sugerem usar as diferenças em desempenho para entender como a informação espacial apresentada na forma dinâmica (ex.: mapas animados) é compreendida e utilizada por indivíduos de diferentes gêneros. Todavia, até o momento, não há resultados que mostrem a relação entre o gênero do motorista e sua demanda visual por mapas dinâmicos exibidos em diferentes escalas, em sistemas de navegação e guia de rota. Sendo assim, o objetivo deste trabalho é avaliar a demanda visual de motoristas dos gêneros masculino e feminino por mapas de SINGRA em diferentes escalas. As questões investigadas são: "Os motoristas do gênero masculino apresentam demanda visual similar aos motoristas do gênero feminino ao interagirem com mapas em diferentes escalas para realizarem diferentes tipos de manobras?"; "A demanda visual gerada nos homens e nas mulheres está relacionada com a variação da escala do mapa?"; e "A demanda visual gerada nos homens e nas mulheres está relacionada com a complexidade da manobra que está sendo representado no mapa?".

\section{MÉTODO}

\subsection{Participantes}

O teste foi realizado com um grupo de 52 motoristas voluntários, 26 homens e 26 mulheres (Tabela 1). Para serem considerados aptos a participarem do teste, os motoristas deveriam possuir: Carteira Nacional de Habilitação $(\mathrm{CNH})$, experiência na direção de automóvel por pelo menos dois anos, visão normal de cores, idade inferior a 40 anos, e pouca ou nenhuma familiaridade com a rota experimental, conforme abordagem utilizada por Ching-Torng et al. (2010), Pugliesi et al. (2009) e Ramos et al. (2014b). Essas informações de caracterização individual foram coletadas por meio de um questionário, o qual foi aplicado em forma de entrevista semiestruturada. Aplicou-se também um teste de habilidade espacial, o teste 
'Tomada de Perspectiva/Orientação Espacial' (Perspective Taking/Spatial Orientation Test - PT/SOT) de Hegarty e Waller (2004).

$\mathrm{O}$ teste PT/SOT é composto por 12 questões sobre a orientação relativa entre 'n' objetos no espaço. A tarefa do indivíduo é imaginar-se de pé, em frente a um objeto de uma cena, olhando para um segundo objeto, e desenhar uma seta que indique a direção de um terceiro objeto. Este teste é recomendado para avaliar a habilidade espacial relacionada a tarefas que envolvem a manipulação mapas (HEGARTY; WALLER, 2004). O intuito da aplicação do teste PT/SOT foi o de determinar a habilidade espacial dos motoristas, em termos de sua habilidade de orientação e, com isso, avaliar se esta característica está relacionada ao gênero ou ao seu desempenho na tarefa de navegação. A habilidade espacial refere-se à capacidade de um indivíduo produzir, representar, transformar e recuperar a informação espacial em sua memória de longa duração (COLUCCIA; LOUSE, 2004).

Tabela 1 - Estatística descritiva.

\begin{tabular}{l|l|l|l|l|l}
\hline \multirow{2}{*}{ Gênero } & \multicolumn{5}{|l}{ Idade dos participantes do teste } \\
\cline { 2 - 6 } & Mínima & Máxima & Média & $\begin{array}{l}\text { Desvio- } \\
\text { padrão }\end{array}$ & Total \\
\hline Masculino & 21 & 37 & 27,5 & 4,83 & 26 \\
\hline Feminino & 22 & 38 & 28,6 & 4,41 & 26 \\
\hline Total médio & 21,5 & 37,5 & 28,05 & 4,62 & Soma = 52 \\
\hline
\end{tabular}

\subsection{Rota Experimental e Simulador de Direção}

A rota é composta por 13 manobras, das quais oito são manobras simples (ex.: manobra à direita, manobra à esquerda), e cinco são manobras complexas (ex.: rotatória). O levantamento dos dados em campo consistiu em filmar a rota experimental e registrar suas coordenadas. A filmagem foi realizada com um telefone celular (marca Iphone 4S), e o levantamento das coordenadas com um GPS Garmin $12 \mathrm{XL}$, no formato NMEA 2.0.

O aparato tecnológico utilizado na avaliação da eficiência das representações cartográficas consistiu em um simulador de direção de baixo custo, com base fixa, pertencente à FCT/UNESP. Para simular uma viagem, o simulador sincroniza a reprodução do vídeo da rota percorrida em campo, com a simulação do deslocamento do automóvel pela rota em um protótipo de SINGRA áudio-dinâmico desenvolvido na FCT/UNESP (PUGLIESI et al., 2009; MARQUES et al., 2012). O recorte da cabina de um automóvel Renault Clio/1991 é usado como veículo. O vídeo da rota é exibido em uma tela de projeção $(2,4 \times 1,8 \mathrm{~m})$, e os mapas do SINGRA em um monitor de sete polegadas (1024x768 pixels). O monitor é posicionado na parte central e superior do painel do automóvel, voltado para a face do motorista, sem obstruir seu campo de visão, conforme recomendações da literatura (WITTMANN et al., 2006). 
O movimento dos olhos do motorista foi registrado pela câmara de um tablet (1024x768 pixels) com resolução de três megapixels, o qual é posicionado atrás do monitor de navegação. A reprodução do som do automóvel e do ambiente externo, registrados em campo, bem como o som do sistema de navegação (beep) é realizada por um par de caixas de som localizadas atrás dos bancos dianteiros do automóvel.

\subsection{Representações Cartográficas em Diferentes Escalas}

As representações foram exibidas em duas escalas: 1:3.000 e 1:6.000, na resolução de $1024 \times 768$ pixels. Estas escalas foram definidas com base nos resultados apresentados por Marques et al. (2012) e Ramos et al. (2014a). O projeto gráfico das representações foi realizado no ArcGIS ArcInfo 10.0. A implementação destas no protótipo de SINGRA foi feito utilizando o compilador Microsoft Visual Basic 6.0 e a biblioteca ESRI Mapobjects 2.1. Nas Figuras 1 e 2 estão algumas das representações projetadas nas duas diferentes escalas para informar a direção em manobras simples e complexa. Estas figuras estão em tamanho cinco vezes menor em relação às imagens apresentadas aos motoristas durante o teste.

Figura 1 - Mapa na escala 1:3.000 (a) e 1:6.000 (b) para a representação de manobra simples.

(a)

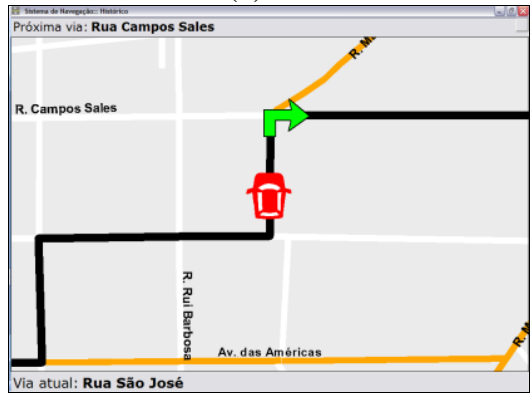

(b)

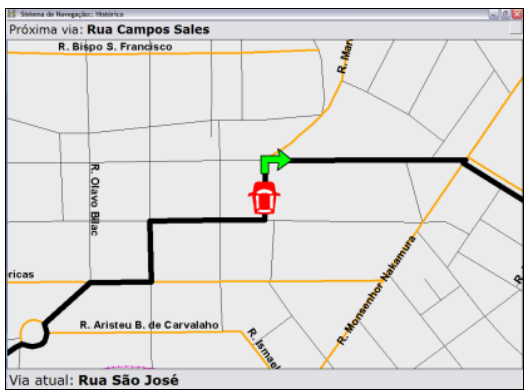

Figura 2 - Mapa na escala 1:3.000 (a) e 1:6.000 (b) para a representação de manobra complexa.

(a)

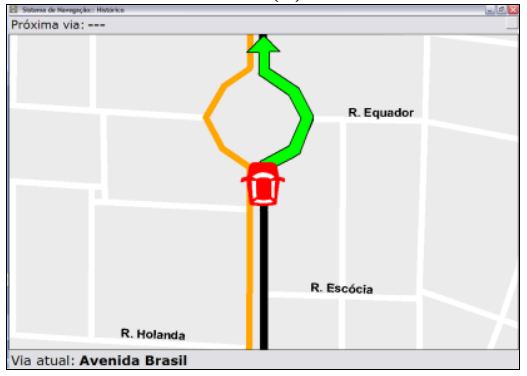

(b)

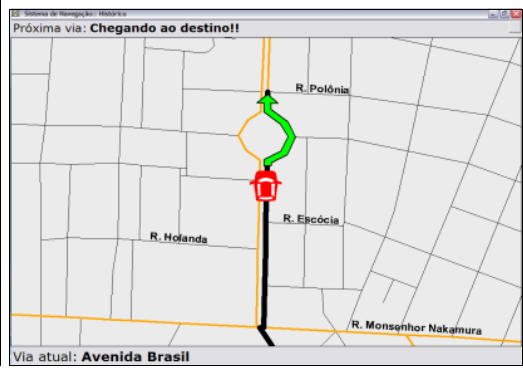

Bol. Ciênc. Geod., sec. Artigos, Curitiba, v. 21, nº 1, p.74-91, jan-mar, 2015. 


\subsection{Aplicação do Teste no Simulador}

$\mathrm{O}$ teste foi iniciado com uma breve explanação da pesquisa e a familiarização do motorista com o simulador de direção. O motorista foi orientado a manusear os controles do veículo (ex.: girar o volante, usar o câmbio de marchas e sinalizadores), porém foi enfatizado que qualquer movimento não alteraria o cenário de simulação. $\mathrm{Na}$ sequência, o motorista fez o preenchimento do questionário de caracterização individual, realizou o teste de orientação espacial e assinou o termo de consentimento. Além disso, o motorista foi familiarizado com o beep e, também, com os mapas nas duas diferentes escalas por meio da apresentação de um 'manual de símbolos' do sistema.

Antes de iniciar a tarefa de simulação da direção, os motoristas foram divididos em dois grupos similares de 13 homens e 13 mulheres cada um. $\mathrm{O}$ intuito foi manter uma aleatoriedade na ordem de exibição das escalas para evitar influências nos resultados de demanda visual. O primeiro grupo iniciou a tarefa de simulação usando mapas em 1:3.000 (manobras de 1 a 6) e finalizou esta tarefa com mapas em 1:6.000 (manobras de 7 a 13). O segundo grupo utilizou a ordem inversa; primeiro os mapas em 1:6.000 (manobras de 1 a 6) e, depois, em 1:3.000 (manobras de 7 a 13).

Durante a realização da tarefa de simulação da direção, o motorista foi orientado a realizar duas tarefas: uma principal e uma secundária. A principal consistiu em prestar a máxima atenção no vídeo da rota, como se estivesse dirigindo o automóvel no mundo real para evitar riscos de acidente no trânsito. A tarefa secundária consistiu em consultar o monitor de navegação, preferencialmente, depois de ouvir o beep, para obter a informação de direção de manobra. O beep foi disparado quando o automóvel se encontrava a cerca de 100 metros ou menos da manobra, representando o início da tarefa tática como abordagem adotada por Pugliesi et al. (2009). A tarefa tática é considerada a etapa mais crítica da navegação do ponto de vista da segurança, porque durante esta tarefa o motorista requer um maior apoio do sistema, resultando em uma maior demanda de processamento mental por parte do usuário (BURNETT, 1998; LABIALE, 2001; KABER et al., 2012).

\subsection{Extração da Demanda Visual}

A caracterização da demanda visual foi realizada a partir de um conjunto de cinco variáveis, quais sejam: número de olhadas, duração mínima da olhada, duração máxima da olhada, duração média da olhada e soma das olhadas. Este conjunto são as variáveis mais importantes no processo de quantificação da demanda visual (TSIMHONI et al., 1999; KLAUER et al., 2006; PUGLIESI et al., 2013). O 'Número de olhadas' (NO) é a quantidade de vezes que uma interface é consultada durante a realização de uma tarefa (TSIMHONI \& GREEN, 2001; KLAUER et al., 2006). A 'Soma das olhadas' (SomaO) representa o tempo total gasto com os olhos fora da via para consultar uma interface (TSIMHONI \& 
GREEN, 2001; KLAUER et al., 2006; PUGLIESI et al., 2013). A 'Duração mínima da olhada' (DurMin) consiste no menor tempo despedido para consultar a informação no mapa. A 'Duração média da olhada' (DurMed) é a soma das olhadas dividida pelo número de olhadas (TSIMHONI et al., 1999), e representa o tempo médio requerido pelo motorista para consultar o mapa. A 'Duração máxima da olhada (DurMax) é a olhada de maior tempo de duração no conjunto de olhadas realizadas para consultar o mapa.

Para extrair os dados de demanda visual, em termos das cinco variáveis quantitativas, adotou-se o método utilizado por Pugliesi et al. (2009) e Ching-Torng et al. (2010). O procedimento consiste em analisar o movimento dos olhos dos motoristas 'quadro a quadro' utilizando um reprodutor de vídeo, neste caso o Microsoft Windows Movie Maker. A comparação da demanda visual entre as diferentes manobras da rota foi realizada para o menor intervalo de tempo de duração da tarefa tática, o qual correspondeu a 11 segundos. Assim, para cada um dos 52 motoristas, nas 13 manobras da rota, analisou-se a demanda visual correspondente ao intervalo de 11 segundos restantes até a manobra.

\section{RESULTADOS}

\subsection{Estatística Descritiva}

Os dados de demanda visual foram organizados no Microsoft Excel e processados no SPSS 16.0 (Statistical Package for the Social Sciences). Para a organização dos dados, considerou-se as escalas (1:3.000 e 1:6.000), o tipo de manobra - Manobra Simples (MS) ou Manobra Complexas (MC), e as cinco variáveis de caracterização da demanda visual - número de olhadas, duração mínima da olhada, duração máxima da olhada, duração média da olhada e soma das olhadas.

Sobre os dados de cada uma das variáveis de caracterização da demanda visual (variáveis dependentes), fez-se a estatística descritiva e aplicou-se o teste de normalidade, no intuito de se definir o tipo de teste estatístico a ser usado nas análises, se paramétrico ou não-paramétrico. Os resultados da estatística descritiva e do teste de normalidade encontram-se apresentados nas Tabelas 2, 3, 4, 5 e 6.

A partir dos resultados do teste de Shapiro-Wilk, aplicado a um nível de confiabilidade de 95\%, observou-se que algumas das variáveis de demanda visual não apresentaram distribuição de probabilidade normal ( $\mathrm{p}$-valor >0,05). Para os conjuntos de dados com distribuição normal, aplicou-se testes não-paramétricos, tais como Wilcoxon e Mann-Whitney U. Para os dados com distribuição desconhecida, aplicou-se testes paramétricos, como teste t para amostras dependentes, e teste t para amostras independentes, conforme recomenda Conover (1999). Quanto aos resultados do teste de orientação espacial, PT/SOT, observou-se que os homens erraram em média $33^{\circ}\left( \pm 34,7^{\circ}\right)$ na estimativa da direção dos objetos, enquanto as mulheres erraram $64^{\circ}\left( \pm 50^{\circ}\right)$. Tais resultados serão discutidos na próxima sessão. 
Tabela 2 - Estatística para a variável 'número de olhadas'.

\begin{tabular}{l|l|l|l|l}
\hline $\begin{array}{l}\text { Tipo de manobra - } \\
\text { escala }\end{array}$ & $\begin{array}{l}\text { Mínimo / } \\
\text { Máximo }\end{array}$ & $\begin{array}{l}\text { Média / Desvio- } \\
\text { padrão }\end{array}$ & Mediana & $\begin{array}{l}\text { Shapiro- } \\
\text { wilk } \\
\text { p-valora }\end{array}$ \\
\hline Simples - 1:3.000 & $1,00 / 7,00$ & $2,72 / 1,23$ & 2,60 & 0,001 \\
\hline Simples - 1:6.000 & $1,00 / 5,00$ & $2,53 / 1,01$ & 2,00 & 0,001 \\
\hline Complexa- 1:3.000 & $1,33 / 5,50$ & $3,22 / 0,95$ & 3,16 & 0,121 \\
\hline Complexa- 1:6.000 & $1,00 / 4,33$ & $2,67 / 0,87$ & 2,58 & 0,137 \\
\hline
\end{tabular}

${ }^{a}$ Assume-se distribuição normal para p-valor superior a 0,05 .

Tabela 3 - Estatística para a variável 'duração mínima da olhada'.

\begin{tabular}{l|l|l|l|l}
\hline $\begin{array}{l}\text { Tipo de manobra - } \\
\text { escala }\end{array}$ & $\begin{array}{l}\text { Mínimo / } \\
\text { Máximo }\end{array}$ & $\begin{array}{l}\text { Média / Desvio- } \\
\text { padrão }\end{array}$ & Mediana & $\begin{array}{l}\text { Shapiro- } \\
\text { wilk } \\
\text { p-valora }\end{array}$ \\
\hline Simples - 1:3.000 & $0,48 / 1,76$ & $0,85 / 0,25$ & 0,80 & $<0,0001$ \\
\hline Simples - 1:6.000 & $0,48 / 2,48$ & $0,87 / 0,31$ & 0,80 & $<0,0001$ \\
\hline Complexa- 1:3.000 & $0,40 / 5,33$ & $2,07 / 1,23$ & 1,96 & $<0,0001$ \\
\hline Complexa- 1:6.000 & $0,58 / 2,68$ & $1,09 / 0,38$ & 1,04 & $<0,0001$ \\
\hline
\end{tabular}

${ }^{a}$ Assume-se distribuição normal para p-valor superior a 0,05 .

Tabela 4 - Estatística para a variável 'duração máxima da olhada'.

\begin{tabular}{l|l|l|l|l}
\hline $\begin{array}{l}\text { Tipo de manobra - } \\
\text { escala }\end{array}$ & $\begin{array}{l}\text { Mínimo / } \\
\text { Máximo }\end{array}$ & $\begin{array}{l}\text { Média / Desvio- } \\
\text { padrão }\end{array}$ & Mediana & $\begin{array}{l}\text { Shapiro- } \\
\text { wilk } \\
\text { p-valora }\end{array}$ \\
\hline Simples - 1:3.000 & $0,64 / 2,37$ & $1,30 / 0,40$ & 1,24 & 0,012 \\
\hline Simples - 1:6.000 & $0,72 / 2,53$ & $1,30 / 0,45$ & 1,20 & $<0,0001$ \\
\hline Complexa- 1:3.000 & $0,88 / 3,40$ & $1,93 / 0,59$ & 1,84 & 0,070 \\
\hline Complexa- 1:6.000 & $0,88 / 4,11$ & $1,75 / 0,60$ & 1,62 & $<0,0001$ \\
\hline
\end{tabular}

${ }^{a}$ Assume-se distribuição normal para p-valor superior a 0,05 .

Tabela 5 - Estatística para a variável 'duração média da olhada'.

\begin{tabular}{l|l|l|l|l}
\hline $\begin{array}{l}\text { Tipo de manobra - } \\
\text { escala }\end{array}$ & $\begin{array}{l}\text { Mínimo / } \\
\text { Máximo }\end{array}$ & $\begin{array}{l}\text { Média / Desvio- } \\
\text { padrão }\end{array}$ & Mediana & $\begin{array}{l}\text { Shapiro- } \\
\text { wilk } \\
\text { p-valora }\end{array}$ \\
\hline Simples - 1:3.000 & $0,59 / 1,79$ & $1,05 / 0,29$ & 1,03 & 0,001 \\
\hline Simples - 1:6.000 & $0,68 / 2,48$ & $1,07 / 0,32$ & 0,97 & $<0,0001$ \\
\hline Complexa- 1:3.000 & $0,74 / 2,66$ & $1,44 / 0,42$ & 1,32 & $<0,0001$ \\
\hline Complexa- 1:6.000 & $0,81 / 3,17$ & $1,40 / 0,42$ & 1,31 & $<0,0001$ \\
\hline
\end{tabular}

${ }^{\mathrm{a}}$ Assume-se distribuição normal para p-valor superior a 0,05 . 
Tabela 6 - Estatística para a variável 'soma das olhada'.

\begin{tabular}{c|c|c|c|c}
\hline $\begin{array}{c}\text { Tipo de manobra - } \\
\text { escala }\end{array}$ & $\begin{array}{c}\text { Mínimo / } \\
\text { Máximo }\end{array}$ & $\begin{array}{c}\text { Média / Desvio- } \\
\text { padrão }\end{array}$ & Mediana & $\begin{array}{c}\text { Shapiro- } \\
\text { wilk } \\
\text { p-valora }\end{array}$ \\
\hline Simples - 1:3.000 & $0,64 / 6,78$ & $2,81 / 1,37$ & 2,52 & 0,002 \\
\hline Simples - 1:6.000 & $0,80 / 5,86$ & $2,67 / 1,20$ & 2,36 & 0,003 \\
\hline Complexa- 1:3.000 & $1,44 / 6,92$ & $4,41 / 1,36$ & 4,21 & 0,500 \\
\hline Complexa- 1:6.000 & $1,44 / 6,46$ & $3,58 / 1,40$ & 3,41 & 0,013 \\
\hline
\end{tabular}

${ }^{a}$ Assume-se distribuição normal para p-valor superior a 0,05 .

\subsection{Demanda Visual e a Relação com o Gênero}

Duas análises foram conduzidas para comparar a demanda visual dos motoristas em relação às representações cartográficas exibidas nas duas escalas, 1:3.000 e 1:6.000. A primeira compara a demanda visual entre os motoristas de diferentes gêneros, homem versus mulher. A partir desta análise, pode-se responder à primeira questão investigada neste trabalho: "Os motoristas do gênero masculino apresentam demanda visual similar aos motoristas do gênero feminino ao utilizarem mapas em diferentes escalas para realizarem manobras simples e complexa?".

A segunda análise compara a demanda visual de motoristas de mesmo gênero para avaliar se a variação da escala do mapa tem maior influência sobre o desempenho das mulheres ou dos homens, na realização da tarefa tática em manobra simples e manobra complexa. A partir desta segunda análise, pode-se responder às outras duas questões investigadas neste trabalho, quais sejam: "A demanda visual gerada nos homens e nas mulheres está relacionada com a variação da escala do mapa?"; e "A demanda visual gerada nos homens e nas mulheres está relacionada com a complexidade da manobra que está sendo representado no mapa?".

\subsubsection{Demanda Visual em Relação a Motoristas de Diferentes Gêneros}

A comparação da demanda visual em função do gênero (Tabela 7) mostrou que as mulheres realizam um maior número de olhadas que os homens, tanto para o mapa exibido em 1:3.000 ( $\mathrm{p}=0,008)$ quanto em 1:6.000 $(\mathrm{p}=0,071)$ em se tratando de manobra simples (Caso A e Caso C). As mulheres também apresentam maior demanda visual comparado aos homens em termos da 'Soma das olhadas' ( $p=$ 0,146 ) ao navegarem com o mapa em 1:3.000. Isto permite afirmar que os motoristas do gênero feminino despendem mais tempo com os olhos fora da via procurando por informação espacial comparado aos motoristas do gênero masculino. Por outro lado, a duração mínima da olhada $(p=0,125)$ e a duração média das olhadas $(\mathrm{p}=0,187)$ realizadas pelos homens é superior ao tempo requerido pelas mulheres para consultarem o mapa em 1:3.000 (Tabela 7).

Em se tratando da tarefa tática em manobra complexa, nenhuma relação foi encontrada entre a demanda visual e o gênero do motorista para o mapa na escala 1:3.000 (Caso B) (Tabela 7). Esta evidência mostra que homens e mulheres têm 
desempenho similar, em termos de demanda visual, ao realizarem manobra complexa quando auxiliados por mapa menos generalizado (1:3.000). Há somente uma tendência $(\mathrm{p}=0,129)$ dos homens apresentarem valor de duração máxima da olhada superior ao das mulheres quando navegam auxiliados pelo mapa em 1:3.000 (Caso D).

Tabela 7 - Demanda visual por mapas em relação ao gênero dos motoristas. TESTE ESTATÍSTICO NÃO-PARAMÉTRICO

\begin{tabular}{|c|c|c|c|c|c|}
\hline \multicolumn{6}{|c|}{ TESTE ESTATISTICO NÃO-PARAMÉTRICO } \\
\hline \multirow{3}{*}{$\begin{array}{l}\text { Tipo de } \\
\text { manobra - } \\
\text { escala }\end{array}$} & \multirow{3}{*}{$\begin{array}{c}\text { Variável de } \\
\text { demanda visual }\end{array}$} & \multicolumn{4}{|c|}{ Teste de Mann-Whitney U (média dos ranks) } \\
\hline & & \multicolumn{2}{|c|}{ Gênero } & \multirow{2}{*}{$\mathrm{Z}$} & \multirow{2}{*}{ p-valor } \\
\hline & & Masculino & Feminino & & \\
\hline \multirow{5}{*}{$\begin{array}{l}\text { Caso A: } \\
\text { Simples - } \\
\text { 1:3.000 }\end{array}$} & NO & 20,94 & 32,06 & $-2,6559$ & $0,008 \mathrm{a}$ \\
\hline & DurMin & 29,71 & 23,29 & $-1,533$ & $0,125 \mathrm{c}$ \\
\hline & DurMax & 27,77 & 25,23 & $-0,605$ & 0,545 \\
\hline & DurMed & 29,27 & 23,73 & $-1,318$ & $0,187 \mathrm{c}$ \\
\hline & SomaO & 23,44 & 29,56 & $-1,455$ & $0,146 \mathrm{c}$ \\
\hline \multirow{3}{*}{$\begin{array}{c}\text { Caso B: } \\
\text { Complexa - } \\
1: 3.000\end{array}$} & DurMin & 24,46 & 28,54 & $-0,971$ & 0,331 \\
\hline & DurMax & 26,40 & 26,60 & $-0,046$ & 0,963 \\
\hline & DurMed & 27,77 & 25,23 & $-0,604$ & 0,546 \\
\hline \multirow{5}{*}{$\begin{array}{l}\text { Caso C: } \\
\text { Simples - } \\
\text { 1:6.000 }\end{array}$} & $\mathrm{NO}$ & 22,79 & 30,21 & $-1,807$ & $0,071 b$ \\
\hline & DurMin & 27,75 & 25,25 & $-0,596$ & 0,551 \\
\hline & DurMax & 26,52 & 26,48 &,- 009 & 0,993 \\
\hline & DurMed & 27,38 & 25,62 & $-0,421$ & 0,674 \\
\hline & SomaO & 23,92 & 29,08 & $-1,226$ & 0,220 \\
\hline \multirow{4}{*}{$\begin{array}{c}\text { Caso D: } \\
\text { Complexa - } \\
1: 6.000\end{array}$} & DurMin & 24,71 & 28,29 & $-0,852$ & 0,694 \\
\hline & DurMax & 29,69 & 23,31 & $-1,520$ & $0,129 \mathrm{c}$ \\
\hline & DurMed & 28,13 & 24,87 & $-0,778$ & 0,437 \\
\hline & SomaO & 27,35 & 25,65 & $-0,403$ & 0,687 \\
\hline & TESTI & TÍSTICO & AMÉTR & & \\
\hline
\end{tabular}

\begin{tabular}{|c|c|c|c|c|}
\hline \multirow[b]{2}{*}{ Comparação: M x F } & \multicolumn{4}{|c|}{ Teste t para amostras independentes } \\
\hline & Média & $\begin{array}{c}\text { Desvio- } \\
\text { padrão }\end{array}$ & $\mathrm{t}$ & p-valor \\
\hline Caso B: Complexa - 1:3.000 & $\begin{array}{c}3,09 \mathrm{x} \\
3,35\end{array}$ & $\begin{array}{c}1,075 \mathrm{x} \\
0,820\end{array}$ & $-0,965$ & 0,339 \\
\hline Caso D: Complexa - 1:6.000 & $\begin{array}{c}2,67 x \\
2,67\end{array}$ & $\begin{array}{c}0,939 \times \\
0,817\end{array}$ & 0,25 & 0,980 \\
\hline \multicolumn{5}{|c|}{ SOMA DAS OLHADAS } \\
\hline \multirow[b]{2}{*}{ Comparação: M x F } & \multicolumn{4}{|c|}{ Teste t para amostras independentes } \\
\hline & Média & $\begin{array}{c}\text { Desvio- } \\
\text { padrão }\end{array}$ & $\mathrm{t}$ & p-valor \\
\hline Caso B: Complexa - 1:3.000 & $\begin{array}{c}4,26 \mathrm{x} \\
4,55\end{array}$ & $1,32 \times 1,42$ & $-0,785$ & 0,436 \\
\hline
\end{tabular}

Fatores de gênero: $\mathrm{M}=$ masculino; $\mathrm{F}=$ feminino. a Significativo a um nível de significância de 1\%, b a 10\% e c Significativo a um nível de significância de $20 \%$. 


\subsubsection{Demanda Visual em Relação a Motoristas de Mesmo Gênero}

Na Tabela 8 e na Figura 3 apresentam-se os resultados obtidos na comparação da demanda visual entre os motoristas de mesmo gênero. Nota-se que, no caso de manobra simples, a variação da escala não influenciou a demanda visual dos motoristas do gênero masculino (Figura 3a) ou feminino (Figura 3b). Neste tipo de manobra, pode-se inferir que ambas as escalas são eficientes para auxiliar motoristas de diferentes grupos. Contudo, no caso de manobra complexa, observa-se que a variação da escala do mapa influencia significativamente o desempenho dos homens e das mulheres. Em pelo menos três variáveis de demanda visual, constatou-se que o mapa em 1:6.000 foi mais eficiente que o mapa em 1:3.000 ao se considerar motoristas de mesmo gênero.

Quanto à comparação do desempenho do motorista em função da variação da complexidade da manobra, tem-se que ambos os grupos de motoristas, homens ou mulheres, apresentaram menor demanda visual ao consultarem o mapa para realizar manobra simples comparado à manobra complexa, independentemente da escala do mapa, 1:3.000 ou 1:6.000. Isso mostra que o aumento na complexidade da manobra influencia na demanda visual, porém este aumento não está correlacionado ao gênero do motorista.

Tabela 3 - Demanda visual para cada tipo de representação cartográfica em relação a motoristas de mesmo gênero.

\begin{tabular}{|c|c|c|c|c|c|c|c|c|}
\hline \multirow{3}{*}{$\begin{array}{c}\text { Demanda visual } \\
\text { (p-valor) }\end{array}$} & \multicolumn{4}{|c|}{ Variação da escala } & \multicolumn{4}{|c|}{ Variação da complexidade da manobra } \\
\hline & \multicolumn{2}{|c|}{$\begin{array}{c}\text { Manobra simples } \\
(1 / 3.000 \mathrm{x} \\
1 / 6.000)\end{array}$} & \multicolumn{2}{|c|}{$\begin{array}{l}\text { Manobra complexa } \\
(1 / 3.000 \times 1 / 6.000)\end{array}$} & \multicolumn{2}{|c|}{$\begin{array}{c}1 / 3.000 \\
(\mathrm{MS} \times \mathrm{MC})\end{array}$} & \multicolumn{2}{|c|}{$\begin{array}{c}1 / 6.000 \\
(\mathrm{MS} \times \mathrm{MC})\end{array}$} \\
\hline & M & $\mathrm{F}$ & $\mathrm{M}$ & $\mathrm{F}$ & $\mathrm{M}$ & $\mathrm{F}$ & M & $\mathrm{F}$ \\
\hline Número de olhadas & 0,840 & $0,158^{\mathrm{d}}$ & $0,017^{b}$ & $0,001^{\mathrm{a}}$ & $0,001^{2}$ & 0,258 & $0,126^{\mathrm{d}}$ & 0,622 \\
\hline Duração mínima & 0,819 & 0,542 & $0,005^{\mathrm{a}}$ & $0,002^{2}$ & $0,001^{a}$ & $<0,0001^{a}$ & 0,201 & $<0,0001^{\mathrm{a}}$ \\
\hline Duração máxima & 0,367 & 0,957 & 0,393 & $0,014^{b}$ & $<0,0001^{2}$ & $<0,0001^{2}$ & $<0,0001^{\mathrm{a}}$ & $0,041^{b}$ \\
\hline Duração média & $0,195^{\mathrm{d}}$ & 0,298 & 0,882 & 0,374 & $<0,0001^{\mathrm{a}}$ & $<0,0001^{2}$ & $<0,0001^{\mathrm{a}}$ & $0,002^{a}$ \\
\hline Soma das olhadas & 0,929 & 0,501 & $0,011^{b}$ & $0,005^{\mathrm{a}}$ & $<0,0001^{\mathrm{a}}$ & $0,001^{\mathrm{a}}$ & $<0,0001^{\mathrm{a}}$ & $0,069^{c}$ \\
\hline
\end{tabular}

Fatores de manobra (MS = Manobra Simples; $M C=$ Manobra Complexa). " p-valor foi estimado usando o teste não-paramétrico de Wilcoxon. ${ }^{*}$ p-valor foi estimado usando o teste paramétrico teste $\mathrm{t}$ para duas amostras relacionadas. ${ }^{a}$ Estatisticamente significativo a um nível de significância de $1 \%$. ${ }^{\mathrm{b}}$ Estatisticamente significativo a um nível de significância de $5 \%$. ${ }^{\mathrm{c}}$ Estatisticamente significativo a um nível de significância de $10 \%$. ${ }^{\mathrm{d}}$ Estatisticamente significativo a um nível de significância de $20 \%$.

Bol. Ciênc. Geod., sec. Artigos, Curitiba, v. 21, nº 1, p.74-91, jan-mar, 2015. 
Figura 3 - Demanda visual dos motoristas do gênero masculino (a) e feminino (b).

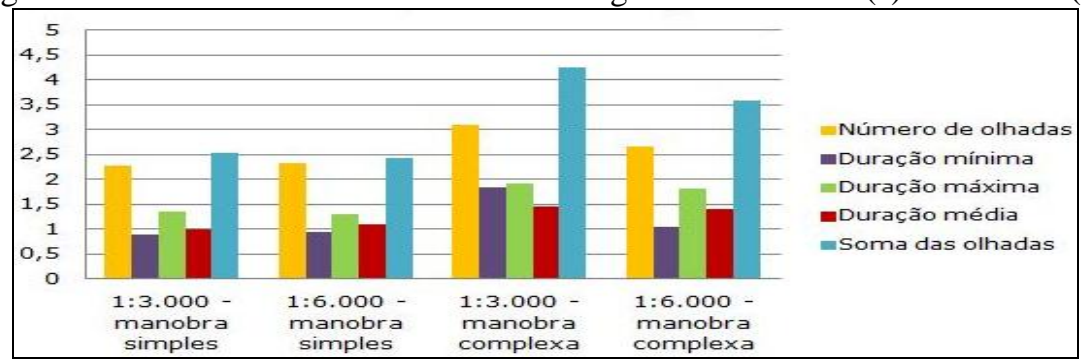

(a)

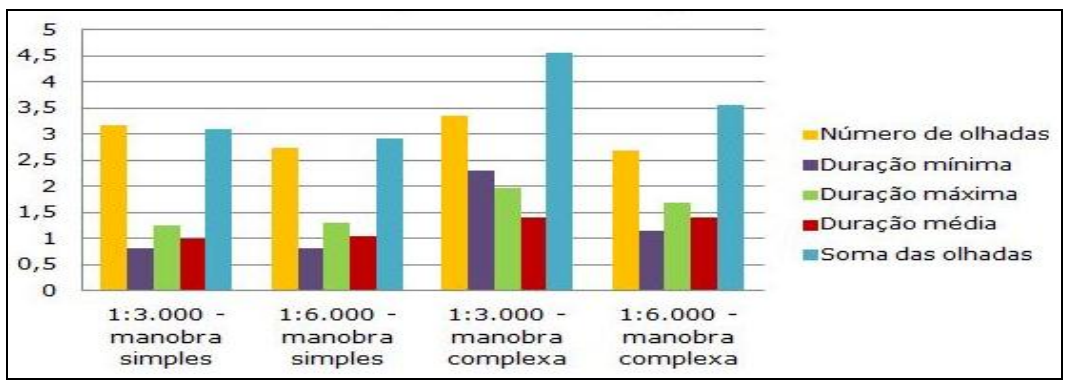

(b)

\section{DISCUSSÃO}

A partir dos resultados obtidos, pode-se afirmar que o gênero é uma característica de grupo que influencia no desempenho do motorista ao realizar tarefas visuo-espacial. Verificou-se que quando auxiliados por mapas em diferentes escalas, para realizarem diferentes tipos de manobras, os motoristas apresentaram variações significativas nos valores de demanda visual. Com base nestas constatações três novas questões foram formuladas: "Por que as mulheres olham mais vezes para o mapa e permanecem mais tempo com os olhos fora da via comparado aos homens?"; "Por que os homens apresentam valor de duração mínima, máxima e de duração média da olhada superior ao realizado pelas mulheres?"; e "Por que as diferenças de demanda visual ocorrem, sobretudo, para o caso de manobra simples visualizada em mapa mais detalhado (escala 1:3.000)?".

$\mathrm{O}$ fato das mulheres consultarem mais vezes o mapa e permanecerem mais tempo com os olhos fora da via, comparado aos homens, evidencia que os motoristas do gênero feminino tem maior necessidade de confirmação se rota está sendo percorrida devidamente. Pugliesi et al. (2009) confirmaram que, comparado aos homens, as mulheres requerem um maior número de olhadas para consultar a informação apresentada em mapa de SINGRA, bem como permanecem mais tempo com os olhos fora da via durante a tarefa tática. Segundo Burnett (1998), a necessidade de confirmação da rota ocorre ao longo de toda a tarefa de navegação, e 
é parte do estágio de confiança. Este estágio é um dos cinco estágios realizados pelos motoristas durante a manutenção em rota, quais sejam estágio de prévisualização, identificação, verificação, confiança e orientação.

Do ponto de vista da psicologia, a necessidade de confirmação da rota demonstrada pelas mulheres pode ser explicada com base no conceito de 'ansiedade espacial'. A ansiedade espacial ou 'o medo de se perder' (KOZLOSWKI \& BRYANT, 1977) é um tipo de sentimento que ocorre ao realizar a tarefa de navegar por um ambiente não familiar (LAWTON, 1994). Estudos mostram que as mulheres utilizam estratégias diferentes comparadas aos homens ao desenvolverem tarefas de navegação em áreas desconhecidas (DABBS et al., 1998; TSIMHONI et al., 1999; WU et al., 2013). Se por um lado os homens se autoclassificam com maior senso de direção e se mostram mais confiantes na sua habilidade em explorar novos caminhos sem o auxílio de um mapa, por outro, as mulheres tendem a apresentar maior nível de ansiedade espacial ao realizarem este mesmo tipo de tarefa (LAWTON, 1994; MONTELLO et al., 1999; COLUCCIA \& LOUSE, 2004; WAKABAYASHI, 2011).

Uma das razões apontadas pela literatura para justificar a maior ansiedade espacial por parte das mulheres é o fato de apresentarem menor habilidade espacial (DABBS et al., 1998; COLUCCIA et al., 2007; WAKABAYASHI, 2011; WILKENING \& FABRIKANT, 2011). Hooven et al. (2004) afirmam que a habilidade espacial é fortemente influenciada pelo nível de testosterona presente no organismo de um indivíduo. Assim, ao se considera que no teste PT/SOT o senso de orientação dos homens foi duas vezes melhor comparado ao das mulheres, parece que tais resultados corroboraram as afirmações de Hooven et al. (2004), porém estudo complementar é necessário para comprovar tal fato.

Em relação ao fato dos homens terem apresentado valores de duração da olhada (mínima, média e máxima) superiores aos das mulheres, este pode ser justificado em razão do baixo número de vezes que o mapa foi consultado. Um experimento conduzido por Ooms et al. (2012) mostrou que o 'tempo de aprendizado com o mapa' foi maior para os homens; porém, uma vez que a informação foi compreendida, estes passaram a 'ignorar' o mapa resultando na redução do número de olhadas para a interface. A partir do instante em que o usuário se familiariza com o mapa, a imagem mental deste é recuperada na memória de trabalho do indivíduo permitindo que as tarefas propostas (ex.: compreender a direção da manobra) sejam resolvidas em menor tempo (OOMS et al., 2012). A redução no tempo ou no número de consulta a uma interface é, portanto, decorrente da redução da carga cognitiva gerada na memória de trabalho do usuário (OOMS et al., 2012).

Quanto à pergunta "Por que as diferenças de demanda visual entre gênero ocorrem, sobretudo, para o caso de manobra simples visualizada em mapa mais detalhado (escala 1:3.000)?", esta pode ser abordada a partir de dois pontos: primeiro, com base no nível de complexidade da manobra e, segundo, com base no nível de detalhamento do mapa. As diferenças de demanda visual entre homens e

Bol. Ciênc. Geod., sec. Artigos, Curitiba, v. 21, nº 1, p.74-91, jan-mar, 2015. 
mulheres foram observadas somente para o caso de manobra simples, porque em manobras complexas os homens, assim como as mulheres, passaram a consultar o mapa mais vezes e por mais tempo para compreenderem a informação de direção. Estes resultados são similares aos obtidos por Labiale (2001) e Pugliesi et al. (2009). Evans e Stevens (1997) ressaltam que a complexidade gráfica de uma interface dificulta o motorista extrair rápida e corretamente a informação de interesse, contribuindo para a distração na tarefa de direção do automóvel.

Em relação ao nível de detalhamento do mapa, Green et al. (1995) afirmam que os motoristas têm dificuldade em ler mapas detalhados enquanto dirigem. Diante disso, tais autores recomendam minimizar a quantidade de feições no mapa, sejam representadas na dimensão linear (ex.: vias, limites administrativos, hidrografia), pontual (ex.: pontos de referência, marcos, etc.) ou textual (ex.: nomes de via, nome de rio, etc.). As recomendações de Green et al. (1995) são corroboradas por pesquisas recentes, as quais verificaram que os usuários têm melhor desempenho ao interagirem com mapas em menor nível de detalhe (LAVIE et al., 2011; LAVIE \& ORON-GILAD, 2013). Um mapa com maior nível de detalhamento pode ser associado a um mapa complexo e que promove o aumento da carga cognitiva na memória de trabalho do motorista. Uma maior carga cognitiva está associada a um aumento no tempo de processamento da informação e, no contexto de SINGRA, isto implica em problemas de eficiência da interface (PUGLIESI et al., 2013).

A redução do desempenho do motorista, em termos de demanda visual, ao interagir com o mapa em 1:3.000 também pode ser explicada em razão da exibição de pouca informação de contexto da rota. Os mapas menos generalizados, embora favoreçam a apresentação das feições em mais detalhes, há uma perda de contexto sobre a área geográfica. A informação de contexto espacial é importante para o estágio de confiança (BURNETT, 1998), pois auxilia o motorista a se manter orientado na rota enquanto navega pela mesma (LEE et al., 2008).

\section{CONCLUSÃO E RECOMENDAÇÕES}

Este trabalho avaliou a influência do gênero do motorista na demanda visual por mapas exibidos em diferentes escalas em sistema de navegação e guia de rota em automóvel. As questões investigadas foram respondidas por meio da análise de um conjunto de variáveis importantes no processo de determinação da demanda visual.

Para os casos estudados, conclui-se que motoristas de distintos gêneros apresentam diferenças em desempenho ao se prepararem para a realização da manobra, dependendo do tipo de manobra a ser realizada e da escala do mapa. Este trabalho confirma os relatos da literatura a respeito de que as mulheres sentem maior ansiedade espacial e possuem menor habilidade espacial que os homens. Tais evidências foram constatadas em termos do aumento do número de olhadas que as mulheres realizaram para o mapa, bem como em termos da soma de tempo que permaneceram com os olhos fora da via. $\mathrm{O}$ fato dos homens terem maior habilidade 
espacial e se sentirem menos ansiosos durante a realização de tarefas visuo-espacial explica o fenômeno de se sentirem mais confiantes ao interagirem com mapas para navegarem em áreas pouco ou não familiares.

Conclui-se que apresentar as instruções de navegação em mapas mais generalizados (ex.: 1:6.000) contribui para a redução da demanda visual do motorista, em razão do mapa oferecer maior contexto espacial. A maior sensação de contexto, possivelmente, aumenta a confiança do motorista na tarefa de navegação apoiada por SINGRA. Conclui-se também que o tipo de manobra é um fator determinante para o desempenho do motorista no processo de leitura do mapa. Ambos, homens e mulheres, apresentaram aumento na demanda visual em tarefas táticas relacionadas a manobras complexas, independentemente da escala de representação.

$\mathrm{Na}$ avaliação do desempenho entre motoristas de um mesmo grupo, há evidências para se concluir que os mapas exibidos nas escalas duas diferentes escalas, 1:3.000 e 1:6.000, são eficientes para auxiliar motoristas homens e motoristas mulheres. Houve variação de desempenho para ambos os grupos de motoristas não somente em função da variável de demanda visual analisada, mas também do tipo de manobra realizada.

Ainda que, para os casos estudados, os mapas se apresentaram eficientes, em termos de demanda visual, para auxiliar motoristas de diferentes grupos, recomenda-se avaliar as interfaces em termos de eficácia e satisfação do usuário. Estas são outras duas classes de medidas necessárias na avaliação da usabilidade de uma interface. Recomenda-se também avaliar a usabilidade do mapa para a mudança automática das escalas.

\section{REFERÊNCIAS BIBLIOGRÁFICAS}

BINFENG, L.; ZHU, K.; ZHANG, W.; WU, A.; ZHANG, X. A comparative study of two wayfinding aids for simulated driving tasks - single-scale and dualscale GPS aids. Behaviour \& Information Technology, p.1-11. 2012.

BURNETT, G. E. "Turn right at the King's Head": Drivers' requirements for route guidance information. PhD Thesis, Loughborough University, UK. 1998.

CHING-TORNG,L.; HSIN-CHIEH, W.; TING-YEN, C. Effects of e-map format and sub-windows on driving performance and glance behavior when using an in-vehicle navigation system. International Journal of Industrial Ergonomics. v. 40, p. 330-336. 2010.

COLUCCIA, E.; LOUSE, G. Gender differences in spatial orientation: A review. Journal of Environmental Psychology. v. 24, p. 329-340. 2004.

COLUCCIA, E.; IOSUE, G.; BRANDIMONTE, M. A. The relationship between map drawing and spatial orientation abilities: A study of gender differences. Journal of Environmental Psychology. v. 27, n. 2, p. 135-144. 2007.

CONOVER, W. J. Practical nonparametric statistics. New York: J. Wiley, 1999. 
DABBS, J. M.; CHANG, E-LEE.; STRONG, R. A.; MILUN, R. Spatial Ability, Navigation Strategy, and Geographic Knowledge Among Men and Women. Evolution and Human Behavior, v. 19, n. 2, p.88-98. 1998.

DILLEMUTH, J.; GOLDSBERRY, K.; CLARKE, K. C. Choosing the scale and extent of maps for navigation with mobile computing systems. Journal of Location Based Services. v. 1, n. 1, p. 46-61. 2007.

FLEMING, A. 2013 - The Year of the Woman Car Buyer: Capture This Powerful \& Ever-Growing. Disponível em <http://www. autoremarketing.com/trends. Acesso em 19 jul. 2014.

FREE PRESS, 2013. The female car buyer - She's invisible - but she buys 30 million cars a year. Disponível em <http://she-conomy.com/ report. Acesso em 09 jul. 2014.

GREEN, P. LEVISON, W.; PAELKE, G.; SERAFIN, C. Preliminary human factors design guidelines for driver information systems. Technical report UMTRI, Ann Arbor, MI, The University of Michigan Transportation Research Institute. 1995.

HEGARTY, M.; WALLER, H. A dissociation between mental rotation and perspective-taking spatial abilities. Intelligence. v. 32, p. 175-191. 2004.

HO, A.; LI, Z. Design of Dynamic Map for Land Vehicle Navigation. The Cartographic Journal. v. 41, n. 3, p. 265-270. 2004.

HOOVEN C. A.; CHABRIS C. F.; ELLISON, P. T.; KOSSLYN, S. M. The relationship of male testosterone to components of mental rotation. Neuropsychologia v. 42 p. 782-790. 2004.

KABER, D. B.; LIANG, Y.; ZHANG, Y.; ROGERS, M. L.; GANGAKHEDKAR, S. Driver performance effects of simultaneous visual and cognitive distraction and adaptation behavior. Transportation Research Part F. v. 15, p.491-501. 2012.

KLAUER, S. G.; DINGUS, T. A.; NEALE, V. L.; SUDWEEKS, J. D. The Impact of Driver Inattention on Near-Crash/Crash Risk: An Analysis Using the 100Car Naturalistic Driving Study Data. Technical report National Highway Traffic Safety Administration - NHTSA. Washington, DC, 2006. Disponível em <http://www.nhtsa.gov/ Research/>. Acesso em 03 jul. 2014

KOZLOSWKI, L. T.; BRYANT, K. J. Sense of direction, spatial orientation, and cognitive maps. Journal of Experimental Psychology: Human Perception and Performance, v. 3, n. 4, p. 590-598, 1977.

LABIALE, G. Visual search and preferences concerning different types of guidance displays. Behaviour \& Information Technology. London: Taylor \& Francis. n. 3, v. 20, p.149-158. 2001.

LAIRD EVANS, J., STEVEN, A. Measures of graphical complexity for navigation and route guidance display. Displays, v.17, n.2, p.89-93, 1997.

LAVIE, T.; ORON-GILAD, T.; MEYER, J. Aesthetics and usability of in-vehicle navigation displays. International Journal of Human-Computer Studies. v. 69, p. 80-99. 2011. 
LAVIE, T.; ORON-GILAD, T. Perceptions of electronic navigation displays. Behaviour \& Information Technology. v. 32, n. 8, p. 800-823. 2013.

LAWTON, C. A. Gender Differences in Way-Finding Strategies: Relationship to Spatial Ability and Spatial Anxiety. Sex Roles. v.30, n. 11/12, p.765-779, 1994.

LEE, J.; FORLIZZI, J.; HUDSON, S. Iterative design of MOVE: A situationally appropriate vehicle navigation system. International Journal of HumanComputer Studies. v. 66, p.198-215. 2008.

MARQUES, A. P. S.; DECANINI, M. M. S.; PUGLIESI, E. A. Sistema de Navegação e Guia de Rota áudio-dinâmico com múltiplas escalas automáticas. Revista Brasileira de Cartografia. v. 1, n. 64, p. 57-68. 2012.

MONTELlO, D. R.; LOVELACE, K. L.; GOLLEDGE, R. G.; SELF, C. M. Sexrelated differences and similarities in geographic and environmental spatial abilities. Annals of the Association of American geographers. v. 89. n. 3, p.515-534. 1999.

OOMS, K.; DE MEYER, P.; FACK, V.; ASSCHE, E. V.; WITLOX, F. Interpreting maps through the eyes of expert and novice users. International Journal of Geographical Information Science. v. 26, n. 10, p. 1773-1788, 2012.

PEI-CHUN, L.; SHANG-I, C. The effects of gender differences on the usability of automotive on-board navigation systems - A comparison of 2D and 3D display. Transportation Research Part F. v.19, p.40-51, 2013.

POOLE, A.; BALL, L. Eye Tracking in Human-Computer Interaction and Usability Research: Current Status and Future. In: GHAOUI, C. Encyclopedia of Human-Computer Interaction. Pennsylvania, USA: Idea Group, 2005.

PUGLIESI, E. A.; DECANINI, M. M. S.; RAMOS, A. P. M.; TSUCHIYA, I. Métodos para Avaliação da Usabilidade de Sistemas de Navegação e Guia de Rota. Revista Brasileira de Cartografia. v. 5, n. 65, p. 571-589. 2013.

PUGLIESI, E. A.; DECANINI, M. M. S.; TACHIBANA, V. M. Evaluation of the Cartographic Communication Performance of a Route Guidance and Navigation System. Cartography and Geographic Information Science. v. 36, n. 2, p.193-207. 2009.

RAMOS, A. P. M.; PUGLIESI, E. A.; DECANINI, M. M. S.; OLIVEIRA, R. F. Escalas dos mapas de sistemas de guia de rota comercial. Revista Brasileira de Cartografia. v.1, n. 66. p.195-207. 2014a.

RAMOS, A. P. M.; PUGLIESI, E. A.; DECANINI, M. M. S. TACHIBANA, V. M. Estudo preliminar da preferência de cor para rota e seta de manobra em mapas de sistemas de guia de rota. Boletim de Ciências Geodésicas. v. 20, n. 2, p. 317-333. 2014b.

RAMOS, A.P.M.; PUGLIESI, E.A.; DECANINI, M.M.S.; OLIVEIRA, R.F.; TACHIBANA, V.M. Avaliação de erros navegacionais em sistema de navegação e guia de rota em automóvel com mapas em diferentes escalas. In: XXVI Congresso Brasileiro de Cartografia 2014c, Gramado, Anais...Gramado: FAURGS, 2014. Disponível em 
<http://www.cartografia.org.br.html> Acesso em 10 out 2014.

SHELEIBY, M.; MALEK, M.R.; ALESHEIKH. Automatic Map Scaling in Car Navigation Systems Using Context-aware Computing. World Applied Sciences Journal. v. 3, p. 101-106. 2008.

SLOCUM, T. A.; BLOK, C.; JIANG, B.; KOUSSOULAKOU, A.; MONTELLO, D. R.; FUHRMANN, S.; HEDLEY, N. R. Cognitive and Usability Issues in Geovisualization. Cartography and Geographic Information Science. v. 28, p.61-75, 2001.

TSIMHONI, O.; YOO, W.; GREEN, P. Effects of Workload and Task Complexity on Driving and Task Performance for In-Vehicle displays as Assessed by visual occlusion. Technical report UMTRI-99-37, Ann Arbor, MI, The University of Michigan Transportation Research Institute. 1999.

TSIMHONI, O.; GREEN, P. Visual demand of driving and the execution of display-intensive in-vehicle tasks. In Proceedings of the human factors and ergonomics society 45th annual meeting, 2001, Santa Monica, California, USA. p. 1586-1590.

UANG, S. T.; HWANG, S. L. Effects on driving behavior of congestion information and of scale of in-vehicle navigation systems. Transportation Research Part F. v. 1, n.11, p.423-438. 2003.

WAKABAYASHI, Y. Gender-related differences in the map use of in-vehicle navigation systems. In Proceedings of the International Cartographic Conference, 2011, Paris, France. p. 1-14.

WILKENING, J.; FABRIKANT, S. I. The effect of gender and spatial abilities on map use preferences and performance in road selection tasks. In Proceedings of the International Cartographic Conference, 2011, Paris, France. p. 232-242.

WITTMANN, M.; KISS, M.; GUGG, P.; STEFFEN, A.; FINK, M.; PÖPPEL, E.; KAMIYA, H. Effects of display position of a visual in-vehicle task on simulated driving. Applied Ergonomics. v. 37, p. 187-199. 2006.

WU, C.; ZHAO, G.; LIN, B.; LEE, J. Navigating a car in an unfamiliar country using an internet map: effects of street language formats, map orientation consistency, and gender on driver performance, workload and multitasking strategy. Behaviour \& Information Technology. v. 32, n. 5, p. 425-437. 2013.

WU, A.; ZHANG, X. Identifying Proper Scales on Digital Maps for In-Vehicle Navigation Systems. In: HCI Part III, San Diego, CA, 2009, p. 262-270

(Recebido em agosto de 2014. Aceito em novembro de 2014). 